\title{
Nosebleeds - The Importance of Taking a History
}

\author{
Maj J D C Bennett
}

BSc, FRCS, DCH, RAMC

Senior Specialist in Otolaryngology

British Military Hospital Rinteln

\section{Dr P L F Giangrande}

BSc, MRCP, MRCPath

Research Fellow, Department of Haemophilia Research

The Royal Free Hospital, London

SUMMARY: Nosebleeds are exceedingly common, and usually idiopathic. Cautery under local anaesthetic to the vessels of the nasal septum is often used successfully as a treatment for the recurrent condition. However, rarely, a nosebleed may be a symptom of a more serious underlying disorder and a suitable history and examination are required.

\section{Introduction}

Epistaxis is a common condition, the majority of cases never reaching the attention of the medical profession. For those who present with bleeding that has not responded to simple digital pressure, control is usually achieved by a nasal pack which is removed after twenty four hours. Persistent bleeding from friable vessels at the anterior end of the nasal septum (Little's area) can be prevented by application of chemical or electrical cautery, though care must be taken as over-enthusiastic use of the cautery can result in necrosis of the septum and the development of a perforation (Fig 1).

\section{Patients}

The following cases seen over a twelve month period at the Ear, Nose and Throat Department of the British Military Hospital Rinteln illustrate some of the problems that may present with a seemingly simple nosebleed.

1. A twenty-five year old man was referred with an epistaxis of several hours duration which could not be controlled, depite the use of adrenaline soaked packs. He had a history of nose bleeds from the age of about ten, bruised easily and found that shaving cuts could bleed for up to an hour. At the age of eight he underwent adenoidectomy and bled excessively afterwards. There was no relevant family history and he did not regularly take aspirin or any other drug. His nose had been cauterised excessively - his estimate was about twenty separate times - and this had left him with a perforation of the nasal septum (Fig 1). A full blood count, including platelet count and morphology, was within normal limits. Prothrombin time (PT) and Kaolin cephalin time (KCCT) were both normal. In view of the history he was referred for specialist haematological advice. Platelet aggregation studied suggest this man suffers from thrombasthenia (Glanzmann's Disease). He has been advised never to take aspirin, as this would further impair platelet activity(1). To date he has not had any further serious problems other than occasional slight

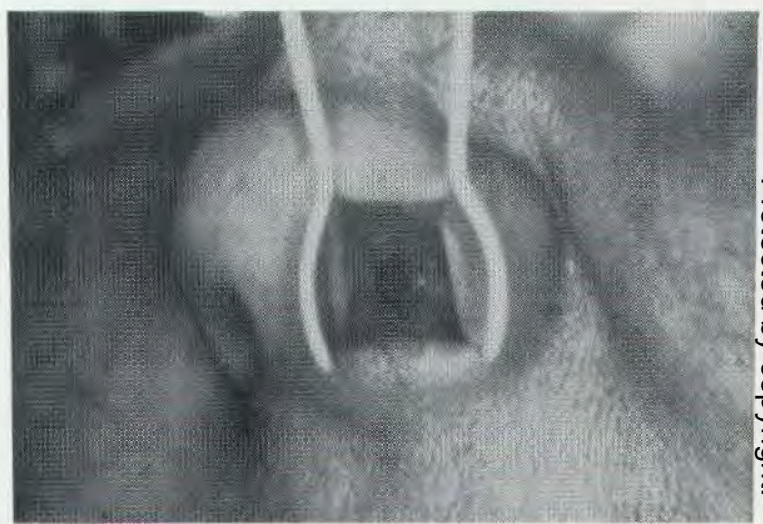

Fig 1. Nasal septal perforation following cautery.

bleeding from the margins of the nasal septal perforation, a recognised complication of this condition.

2. A twenty-eight year old woman presented with epistaxis, controlled only with some difficulty by nasal packing. There was no relevant past medical history and she was not taking any medication. She was noted to have petechiae and thrombocytopaenia was suspected. Her platelet count was $8 \times 10^{\circ} /$. She was referred to a haematologist. After a bone-marrow aspirate the diagnosis of autoimmune thrombocytopaenia was made and treatment with steroids was commenced. In this case the combination of abnormal physical findings and simple laboratory investigations showed that something more serious than a simple nosebleed was present and prompt appropriate referral could be made.

3. A six year old girl was referred on four separate occasions with recurrent nose bleeds. She had cautery to the nasal septum performed several times under local anaesthesia. On closer questioning it was found that she bruised easily and when a tooth fell out she bled for 24 hours. She has one brother, aged eight, who bruises 
easily and her mother, who is adopted and has not traced her natural parents, bruises easily and has heavy periods. The blood count, including platelet count, was normal. The PT was normal but the KCCT was prolonged at 60 seconds (normal 45 ). The bleeding time (BT) was prolonged at 14 minutes.

She was referred for a haematological opinion and further investigations revealed moderately low factor VIII antigen levels as well as impaired aggregation of her platelets with ristocetin. A diagnosis of von Willebrand's disease was therefore made. In severe cases of this disease cryoprecipitate or Factor VIII infusion is necessary to control bleeding. In less severe cases an infusion of desmopressin can be used(2). This is a synthetic analogue of vasopressin which increases the plasma concentration of the Factor VIII thus normalising the haematostatic defect. Tranexamic acid is given in addition to inhibit fibrinolysis.

4. A fifty-two year old man presenting with epistaxis was found to have recently started treatment with oral anticoagulants. His nose was packed and he was given vitamin $\mathrm{K}_{1} 10 \mathrm{mg}$ intravenously. His warfarin was stopped and he was referred back to his anticoagulant clinic. When the prothrombin time or thrombotest is below the generally accepted safe level and reversal is necessary it can usually be achieved by the oral administration of $5 \mathrm{mg}$ vitamin $\mathrm{K}_{1}$ or an intramuscular injection of 1-2 mg. All bleeding should be treated with slow intravenous vitamin $\mathrm{K}_{1}, 25 \mathrm{mg}$ at a rate not exceeding $5 \mathrm{mg}$ per minute. This will take some hours to achieve reversal of the anticoagulant effect. Immediate replacement of vitamin $\mathrm{K}$ dependent factors with infusion of fresh frozen plasma may be necessary, after consultation with a haematologist, preferably the one who commenced anti-coagulation.

\section{Discussion}

In only a small number of cases can epistaxis be attributed to a well defined primary cause such as a blood dyscrasia, a coagulation defect or local nasal pathology(3). The so-called idiopathic group includes about $85 \%$ of patients(4).

In a normal person the response to epithelial damage is aggregation of platelets to form a haemostatic plug at that site. This reaction alone may be enough to arrest haemorrhage even in patients with disorders of the coagulation cascade. For example the bleeding time is entirely normal in haemophilia. The reaction will be defective, however, if there is thrombocytopaenia or defective function of platelets despite a normal platelet count.

In addition to the formation of the platelet plug, the coagulation cascade is activated and this leads to the conversion of soluble fibrinogen into insoluble fibrin strands which form a solid clot. Figure 2 illustrates our current understanding of the cascades(5). The intrinsic pathway is activated by contact of Factor XII on the collagen of exposed subendothelial tissue. The extrinsic pathway involves the direct activation of Factor VII by az tissue thromboplastin which is released from damaged $\mathbb{\Phi}$ tissues. The two arms of the cascade converge on Factor $X$, the activation of which leads to thrombin activity and $O$ subsequent conversion of fibrinogen to fibrin.

A clue to the nature of the bleeding disorder can often be obtained from the history, which should be sought $\stackrel{\vec{P}}{\stackrel{9}{9}}$ diligently with particular attention to problems amongst으 other family members and to drug ingestion. Problems of platelet function tend to produce purpuric $\frac{\text { D }}{\vec{D}}$ haemorrhages while disorders of clotting cause a more $\mathbb{\complement}$ delayed failure to heal after a bleed (the initial platelet $\cong$ plug is formed normally).

Basic screening tests indicate the major defects. A full blood count will reveal a low platelet count. $\overrightarrow{\mathrm{\omega}}$ Examination of a film will reveal the morphology of the platelets and leukocytes and may give a clue to the cause of the thrombocytopenia. Polycythemia, shown by a? high haematocrit, causes bleeding by bursting blood $\stackrel{i}{\omega}$ vessels. The PT measures the activity of the external pathway and is normally 13-16 seconds, being

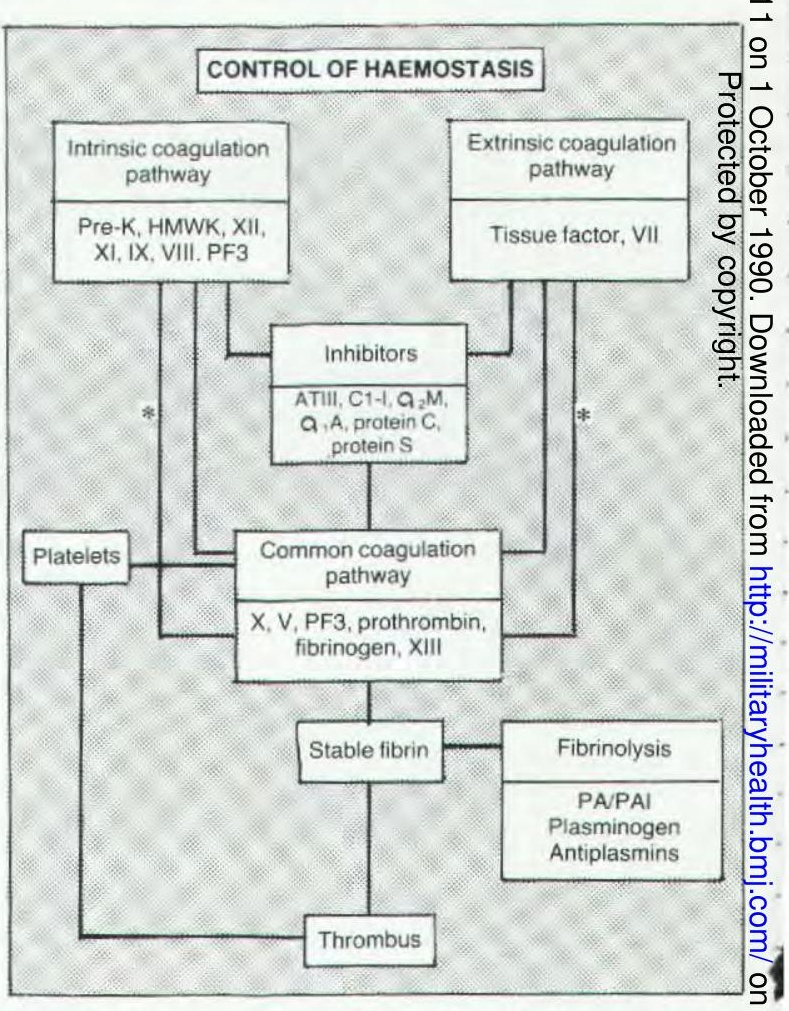

Fig 2. Thrombus formation. *Feedback acceleration within and from common pathway. Pre-K = prekallikrein; HMWK = high molecular weight kinnogen; PF? $=$ platelet factor 3 ; ATIII $=$ antithrombin III; C1-I $=$ C I N inhibitor; $a_{2} \mathbf{M}=a_{2}$-macroglobulin; $a_{1} A=a_{1}$ 足 antitrypsin; $\mathbf{P A} / \mathbf{P A 1}=$ plasminogen activators and $\omega$ inhibitors. 
prolonged by anticoagulants such as warfarin. The KCCT measures the internal pathway and is prolonged from its normal value of $37-45$ seconds by heparin. The bleeding time is measured by applying a tourniquet to a pressure of $40 \mathrm{~mm}$ mercury and making two incisions in the skin with a special apparatus. The cuts are blotted at 30 second intervals and the time taken for haemostatis measured. No aspirin should be taken for seven days beforehand as this interferes with platelet function.

\section{Conclusion}

Haematological disorders can present in a variety of ways to a variety of clinicians, so an understanding and awareness of these conditions is important. This is especially the case in hospitals where specialist haematologist advice "on site" is limited. Adequate preliminary investigations can sometimes clarify the problem, making the process and priority of referral easier. The limitations of these tests should be understood.

\section{Acknowledgement}

The help and advice of Dr Brian M Williams, Consultant Pathologist, British Military Hospital Hannover is gratefully acknowledged.

\section{REFERENCES}

1. SUtor A H, Bowie E J, OWen C A. Effect of Aspirin, Sodium Salicylate \& Acetomorphin on Bleeding. Mayo Clin Proc 1971; 46: 178-181.

2. Blomback M, Johansson G, Johnsson H, SwEdenborg J, WABO E. Surgery in Patients with von Willebrand's Disease. BrJ Surg 1989; 76: 398-400.

3. KERR A G. Scott Brown's Textbook on Otolaryngology. London: Butterworths, 1987.

4. Maran A G D, Stell. P M. Clinical Otolaryngology. London: Blackwell Scientific Publications 1979.

5. Prentice a G, Bradford C R, Copplestone J A. Control of Haemostasis. BrJ Hosp Med 1990; 43: 385-389. Reproduced with permission. 